\title{
COMUNICAÇÃO
}

\section{AVALIAÇÃO DO DESENVOLVIMENTO DA CULTURA DA SOJA (Glycine max (L.) Merrill) SOB DIFERENTES SISTEMAS DE PREPARO}

\author{
Evaluation of the development of soybean crop (Glycine $\max$ (L.) Merrill) \\ under different soil tillage
}

\author{
Gustavo Naves dos Reis ${ }^{1}$, Alexandre Cesar Bizzi ${ }^{2}$, Carlos Eduardo Angeli Furlani ${ }^{3}$, Rouverson Pereira da Silva ${ }^{3}$, \\ Afonso Lopes ${ }^{3}$, Danilo Cesar Checchio Grotta ${ }^{1}$
}

\begin{abstract}
RESUMO
Atualmente na agricultura, a soja ocupa lugar de destaque garantido pela alta produtividade e excelente rentabilidade no mercado nacional, principalmente na exportação de grãos. Sabe-se que para uma boa produtividade é necessário um bom planejamento, além de técnicas de preparo do solo adequadas a cada região do País. Este trabalho, desenvolvido na Universidade Estadual Paulista, Faculdade de Ciências Agrárias e Veterinárias, Campus de Jaboticabal, SP, teve por objetivo, analisar o efeito de diferentes sistemas de preparo no solo e na cultura da soja (Glycine max (L.) Merrill) em Latossolo Vermelho Eutroférrico. Os tratamentos foram 3 preparos convencionais (arado de discos + duas passadas de grade leve, arado de aivecas + duas passadas de grade leve, grade pesada + grade leve), 2 preparos reduzidos (escarificador com rolo destorroador + grade leve, escarificador com rolo destorroador) e semeadura direta, resultando em 6 tratamentos com 4 repetições, totalizando 24 observações. O teor de água no solo não difere entre tratamentos. Na camada superficial $(0-5 \mathrm{~cm})$ a semeadura direta apresenta maior resistência à penetração em relação ao arado de aivecas com duas gradagens leves e escarificador com uma gradagem leve. As características agronômicas da cultura da soja não são influenciadas pelos tratamentos estudados.
\end{abstract}

Termos para indexação: Semeadura direta, cultivo mínimo, resistência à penetração, Glycine max.

\begin{abstract}
In modern agriculture the soy has a place of prominence, which is guaranteed for the high productivity, excellent yield in the market and mainly in the exportation of grains. It's known that in order to achieve a good produce an adequate plan of production and adequate techniques on the preparation of the soil according to its region of the country. This work was developed in the Universidade Estadual Paulista, Faculdade de Ciências Agrárias e Veterinárias, Campus de Jaboticabal, SP, with the objective of analyze the effect of different soil tillage in the soybean crop (Glycine max (L.) Merrill) in a Oxisoil, being they conventional (disc plow + two soft harrowings, moldboard plow and two soft harrowings, heavy disc harrow + soft harrowing), 2 reduced (chiselplow + softharrowing, chiselplow only) and no tillage, resulting in 6 treatments with 4 repetitions. The water level in the ground did not have significant differences between the treatments. In the superficial layer $(0-5 \mathrm{~cm})$ the no tillage presented greater resistance to the penetration in relation to the treatments mondboard plough and two harrowings and chiselplow and harrow. The agronomical characteristics of the soybean crop are not influenced by the studied treatments.
\end{abstract}

Index terms: No tillage, reduced tillage, resistance to the penetration, Glycine max.

\section{(Recebido para publicação em 15 de julho de 2005 e aprovado em 5 de abril de 2006)}

Há cerca de cinco mil anos a soja começou a ser domesticada pelos chineses e apenas no início do século XX passou a ser cultivada comercialmente nos Estados Unidos, alcançando um rápido crescimento na produção, com o desenvolvimento das primeiras cultivares comerciais. No Brasil o grão chegou com os primeiros imigrantes japoneses em 1908, porém, sua expansão aconteceu nos anos 70, com o interesse crescente da indústria de óleo e o aumento da demanda no mercado internacional.
De acordo com Furlani (2000), cada vez mais procura-se o desenvolvimento de novas tecnologias, principalmente relacionadas a equipamentos, para aumentar a eficiência dos mesmos. Porém, em grande parte dos casos, não há preocupação com a qualidade das operações agrícolas, e principalmente, com a conservação dos recursos naturais.

A mecanização agrícola é um importante componente básico na maioria das estratégias de desenvolvimento rural, aumento da produtividade e mão-

\footnotetext{
Engenheiro Agrônomo M.Sc., Doutorando em Agronomia - Departamento de Engenharia Rural - FCAV/UNESP - Via de Acesso Prof. Paulo Donato Castellane s/n - 14.884-900 - Jabotical, SP - gnrunesp@hotmail.com

${ }^{2}$ Engenheiro Agrônomo, formado pela FCAV/UNESP - Via de Acesso Prof. Paulo Donato Castellane s/n - 14.884-900 - Jaboticabal, SP.

${ }_{3}^{3}$ Professor Dr., Departamento de Engenharia Rural da FCAV/UNESP - Via de Acesso Prof. Paulo Donato Castellane s/n - 14.884-900 - Jaboticabal, SP.
} 
de-obra. No entanto, sua introdução maciça, sem qualquer adaptação prévia aos diferentes tipos de solo pode ocasionar rápida e contínua degradação do recurso natural.

O sistema de semeadura direta, cada vez mais presente na agricultura brasileira, é um método que visa maior conservação do solo, e diminuição do tráfego de máquinas possuindo como princípio a semeadura diretamente no solo onde apenas a linha de plantio é revolvida pelos discos de corte ou hastes da semeadora. Porém, hoje ainda é muito utilizado o preparo convencional o qual é composto por aração com arados ou grade aradora, seguido de gradagens niveladoras. Intermediário aos dois sistemas anteriormente citados aparece o preparo reduzido que implica em redução das operações do preparo do solo. Esse sistema já bastante difundido no País é realizado com o uso de escarificadores, normalmente equipados com discos de corte à frente de cada haste, para trabalhos em solos com palha sobre a superfície. Também pode conter o equipamento, um rolo destorroador que tem por função diminuir o tamanho dos torrões e nivelar o solo para a semeadura (BOLLER, 2003).

Visando analisar alguns fatores que podem interferir na produtividade da soja, desenvolveu-se este trabalho que teve por objetivo avaliar possíveis diferenças entre sistemas de preparo em características do solo e no desenvolvimento da cultura da soja (Glycine max (L.) Merrill) em Latossolo Vermelho Eutroférrico.

$\mathrm{O}$ experimento foi instalado e conduzido em janeiro de 2003 na área experimental do Departamento de Engenharia Rural, da Universidade Estadual Paulista, Faculdade de Ciências Agrárias e Veterinárias, Campus de Jaboticabal - SP, localizada nas coordenadas geográficas $21^{\circ} 15^{\prime} \mathrm{S}$ e $48^{\circ} 18^{\prime} \mathrm{O}$, com altitude média de 595 metros e clima Cwa (subtropical), de acordo com a classificação de Köeppen. O solo da área experimental, de acordo com a classificação da Embrapa (1999), corresponde ao Latossolo Vermelho Eutroférrico, de relevo suave ondulado.

O delineamento experimental foi em blocos ao acaso com 6 tratamentos e 4 repetições. Cada parcela, ocupava uma área de $200 \mathrm{~m}^{2}$ (20 x 10 m) e entre elas, na direção longitudinal, reservou-se uma área de $150 \mathrm{~m}^{2}(15$ x $10 \mathrm{~m})$, destinada à realização de manobras.

Utilizou-se 6 tratamentos dos quais, 3 de preparo convencional do solo, 2 de preparo reduzido e 1 com semeadura direta sobre a palha, sendo eles:

T1 - Arado de discos + duas passadas de grade leve (AD+GL);

$\mathrm{T} 2$ - Arado de aivecas + duas passadas de grade leve (AA+GL);
T3 - Grade pesada + grade leve (GP+GL);

T4 - Escarificador com rolo destorroador + grade leve $(\mathrm{ES}+\mathrm{GL})$;

T5 - Escarificador com rolo destorroador (ES);

T6 - Semeadura direta (SD);

A determinação da fertilidade do solo, nas camadas de $0-10 \mathrm{~cm}$ (Tabela 1) e $10-20 \mathrm{~cm}$ (Tabela 2) foi feita pelo Laboratório de Análise de Solo e Plantas, do Departamento de Solos e Adubos da Faculdade de Ciências Agrárias e Veterinárias da UNESP/Jaboticabal - SP. Determinou-se a fertilidade em duas camadas devido à diferença de material orgânico sobre o solo na semeadura direta, quando comparada aos outros tratamentos. Entretanto, para a recomendação da adubação utilizou-se a média das análises químicas do solo obtidas nas duas camadas.

Para a instalação do experimento, foram utilizados $60 \mathrm{~kg} \mathrm{ha}^{-1}$ de sementes de soja, marca Agromen, cultivar Conquista com poder germinativo de $85 \%$ e pureza de $99 \%$.

Utilizou-se $350 \mathrm{~kg} \mathrm{ha}^{-1}$ de adubo na fórmula 4-2020, na semeadura da soja. No preparo dos tratamentos présemeaddura da soja, não se utilizou de herbicida e o manejo da cobertura foi executado com triturador de palhas.

Utilizou-se 3,0 L ha-1 de gliphosate Roundup em pré-emergência para controle de plantas daninhas;

Os tratores e equipamentos utilizados na condução do experimento foram os seguintes:

- Trator 4 x 2 com potência de 55,2 kW (75 cv) no motor, utilizado para as operações de gradagem e pulverizações;

- Trator 4 x 2 TDA com potência de 77,0 kW (105 cv) no motor, utilizado para as operações de aração e tracionamento da semeadora-adubadora;

- Trator 4 x 2 TDA com potência de 110,0 kW (150 cv) no motor, utilizado para as operações de escarificação

- Colhedora de parcelas com potência de $63 \mathrm{~kW}$ $(85 \mathrm{cv})$ e largura útil de 1,0 metro.

- Semeadora-adubadora de arrasto, com 4 linhas espaçadas em $0,45 \mathrm{~m}$, com sulcadores de discos duplos para sementes e sulcador tipo haste para fertilizantes, mecanismo dosador de sementes à vácuo, equipada com rodas compactadoras e calibrador de profundidade;

- Pulverizador de barras montado, com tanque de capacidade de $600 \mathrm{~L}$ de calda, barras equipadas com 24 bicos de jato em leque, com largura útil de $12 \mathrm{~m}$;

- Arado de discos reversível de 3 discos de 26 polegadas;

- Arado de aivecas reversível de 2 aivecas recortadas;

- Grade niveladora de 32 discos de 18 polegadas, com massa aproximada de $1.200 \mathrm{~kg}$; 


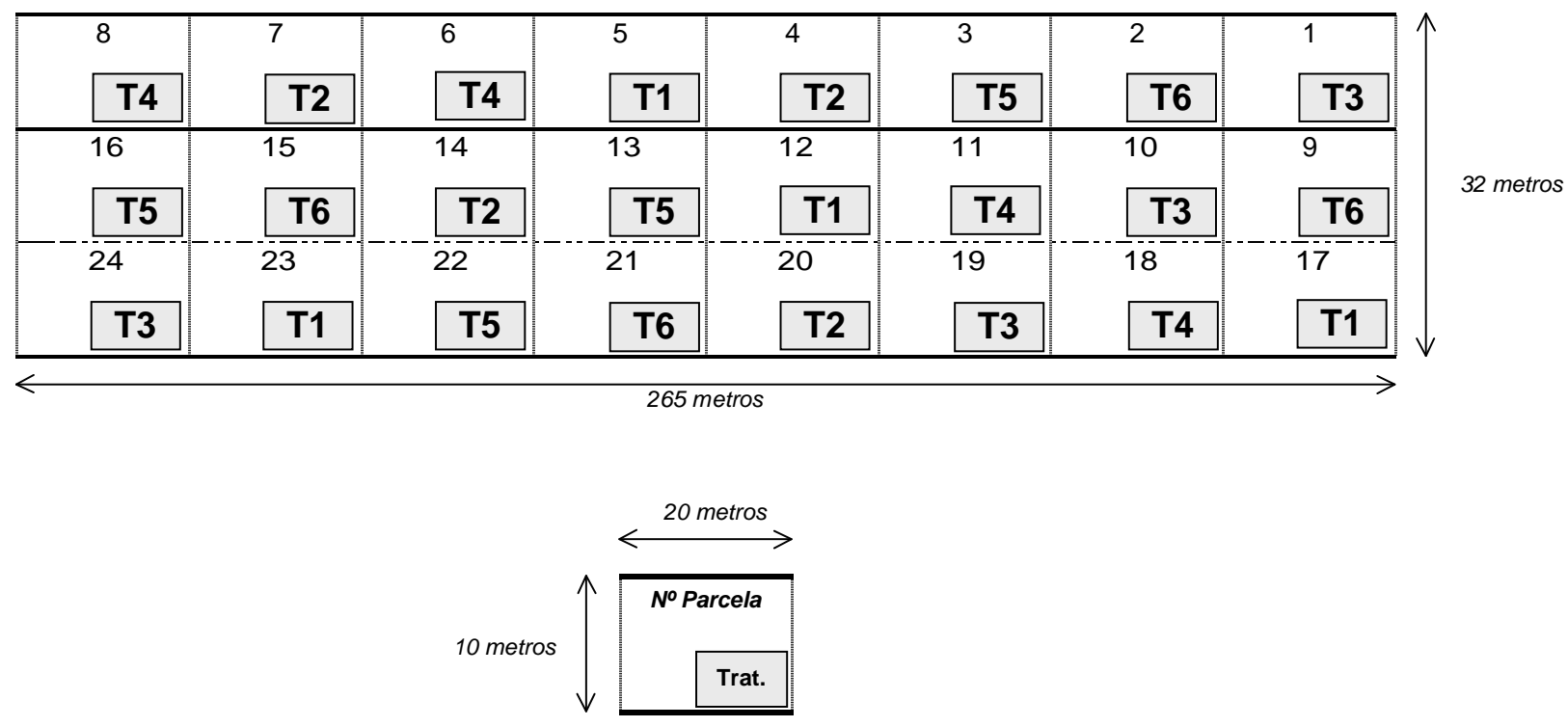

FIGURA 1 - Croqui da área experimental.

TABELA 1 - Resultados da análise química do solo na camada de $0-10 \mathrm{~cm}$ de profundidade, em cada tratamento.

\begin{tabular}{|c|c|c|c|c|c|c|c|c|c|c|}
\hline Tratamentos & $\begin{array}{c}\mathrm{pH} \\
\mathrm{CaCl}_{2}\end{array}$ & $\begin{array}{l}\text { M.O. } \\
\text { g dm }^{-3}\end{array}$ & $\begin{array}{l}\text { P resina } \\
\mathrm{mg} \mathrm{dm}^{-3}\end{array}$ & $\mathbf{K}$ & $\mathbf{C a}$ & $\begin{array}{l}\mathrm{Mg} \\
--\mathrm{m}\end{array}$ & $\begin{array}{l}\mathbf{H}+\mathbf{A l} \\
\mathrm{ldm}^{-3}\end{array}$ & SB & $\mathbf{T}$ & $\begin{array}{l}\mathrm{V} \\
\%\end{array}$ \\
\hline $\mathrm{T} 1-\mathrm{AD}+\mathrm{GL}$ & 5,2 & 26 & 32 & 4,4 & 22 & 8 & 34 & 34,4 & 68,4 & 50 \\
\hline $\mathrm{T} 2-\mathrm{AA}+\mathrm{GL}$ & 5,3 & 26 & 45 & 6,2 & 23 & 10 & 31 & 39,2 & 70,2 & 56 \\
\hline $\mathrm{T} 3-\mathrm{GP}+\mathrm{GL}$ & 5,5 & 27 & 40 & 5,3 & 31 & 11 & 28 & 47,3 & 75,3 & 63 \\
\hline $\mathrm{T} 4-\mathrm{ES}+\mathrm{GL}$ & 5,4 & 27 & 36 & 4,6 & 35 & 13 & 31 & 52,6 & 83,6 & 63 \\
\hline T5 - ES & 5,3 & 27 & 45 & 3,8 & 22 & 8 & 31 & 33,8 & 64,8 & 52 \\
\hline $\mathrm{T} 6-\mathrm{SD}$ & 5,3 & 26 & 29 & 4,1 & 23 & 8 & 31 & 35,1 & 66,1 & 53 \\
\hline
\end{tabular}

TABELA 2 - Análise química do solo na camada de 10-20 cm de profundidade, em cada tratamento.

\begin{tabular}{|c|c|c|c|c|c|c|c|c|c|c|}
\hline Tratamentos & $\underset{\mathrm{CaCl}_{2}}{\mathrm{pH}}$ & $\begin{array}{l}\text { M.O. } \\
\text { g dm}^{-3}\end{array}$ & $\begin{array}{l}\text { P resina } \\
\text { mg dm }^{-3}\end{array}$ & $\mathbf{K}$ & $\mathbf{C a}$ & $\begin{array}{l}\text { Mg } \\
--m m o\end{array}$ & $\begin{array}{r}\mathbf{H}+\mathbf{A l} \\
\mathrm{dm}^{-3}-\ldots\end{array}$ & SB & $\mathbf{T}$ & $\begin{array}{l}\mathrm{V} \\
\%\end{array}$ \\
\hline $\mathrm{T} 1-\mathrm{AD}+\mathrm{GL}$ & 5,1 & 24 & 24 & 2,2 & 20 & 6 & 34 & 28,2 & 62,2 & 45 \\
\hline $\mathrm{T} 2-\mathrm{AA}+\mathrm{GL}$ & 5,1 & 22 & 23 & 2,4 & 19 & 7 & 34 & 28,4 & 62,4 & 46 \\
\hline $\mathrm{T} 3-\mathrm{GP}+\mathrm{GL}$ & 5,4 & 21 & 22 & 3,1 & 26 & 9 & 28 & 38,1 & 66,1 & 58 \\
\hline $\mathrm{T} 4-\mathrm{ES}+\mathrm{GL}$ & 5,4 & 26 & 37 & 3,8 & 29 & 9 & 31 & 41,8 & 72,8 & 57 \\
\hline $\mathrm{T} 5-\mathrm{ES}$ & 5,2 & 25 & 25 & 3,2 & 22 & 8 & 34 & 33,2 & 67,2 & 49 \\
\hline $\mathrm{T} 6-\mathrm{SD}$ & 5,2 & 21 & 18 & 2,5 & 23 & 8 & 34 & 33,5 & 67,5 & 50 \\
\hline
\end{tabular}

Ciênc. agrotec., Lavras, v. 31, n. 1, p. 228-235, jan./fev., 2007 
- Grade aradora de 18 discos de 24 polegadas, com massa aproximada de $2.300 \mathrm{~kg}$;

- Escarificador de 7 hastes com rolo destorroador.

Para a determinação do teor de água do solo, foram feitas duas amostragens por parcela em datas diferentes, sendo que uma amostragem ocorreu na época do florescimento da cultura e a outra após a colheita da mesma nas profundidades de $0-10$ e $10-20 \mathrm{~cm}$. As amostras foram acondicionadas em recipientes de alumínio com volume de $60 \mathrm{~cm}^{3}$, vedadas, pesadas em balança com precisão de $0,01 \mathrm{~g}$ e levadas até o laboratório para secagem em estufa elétrica por um período de 48 horas a $60^{\circ} \mathrm{C}$, para novamente serem pesadas.

Para a determinação da resistência do solo à penetração, utilizou-se penetrômetro de impacto, modelo IAA/Planalsucar-Stolf (STOLF et al., 1983). Foram determinados 3 pontos aleatórios em cada parcela na primeira amostragem e 2 pontos na segunda amostragem nas profundidades de 0 a $40 \mathrm{~cm}$, sendo as camadas analisadas a cada $5 \mathrm{~cm}$. Após obtenção do número de impactos, estes foram transformados em MPa por meio da equação desenvolvida por Stolf (1990).

Para avaliação da população inicial e final das plantas, utilizou-se régua de madeira, com 2 metros de comprimento, colocada no sentido da linha de plantio e contou-se a quantidade de plantas neste segmento. Foram feitas três contagens em diferentes pontos escolhidos aleatoriamente em cada parcela, tanto para estande inicial quanto para final.

Para avaliação da altura das plantas, foram feitas 3 amostras por parcela em pontos aleatórios aos 60 e 90 dias após a semeadura utilizando-se régua de madeira com fita métrica fixada de 1 metro de comprimento colocada em sentido vertical, próximo às hastes principais das plantas, onde as leituras foram tomadas.

Foi determinado o número de vagens por planta e grãos por vagem, colhendo-se cinco plantas por parcela e a determinação do número de grãos por vagem foi obtida através da divisão do número total de grãos pelo número de vagens cheias.

Para a determinação do rendimento da cultura, utilizou-se a colhedora de parcela com plataforma de corte de 1 metro de largura. Os grãos colhidos foram trilhados e separados da palha, sendo sua massa determinada para um teor de água de $11 \%$.

Os dados foram submetidos à análise de variância e ao teste de comparação de médias de Tukey a 5\% de probabilidade por meio do Programa ESTAT desenvolvido pelo Departamento de Ciências Exatas da FCAV/UNESP.
Os resultados obtidos estão apresentados em Tabelas, onde médias seguidas de mesmas letras maiúsculas nas colunas não diferem estatisticamente entre si, pelo teste de Tukey, a 5\% de probabilidade.

Quanto ao teor de água do solo não foram observadas diferenças entre os tratamentos, nas amostragens realizadas (Tabelas 3 e 4).

TABELA 3 - Teor de água do solo (\%) - $1^{\text {a }}$ amostragem*

\begin{tabular}{lcc}
\hline Tratamento & \multicolumn{2}{c}{ Profundidade $(\mathbf{c m})$} \\
\hline T1 - AD+GL & $21,4 \mathrm{~A}$ & $10-20$ \\
T2 - AA+GL & $21,3 \mathrm{~A}$ & $20,1 \mathrm{~A}$ \\
T3 - GP+GL & $21,1 \mathrm{~A}$ & $19,8 \mathrm{~A}$ \\
T4 - ES+GL & $21,3 \mathrm{~A}$ & $19,9 \mathrm{~A}$ \\
T5 - ES & $22,2 \mathrm{~A}$ & $21,0 \mathrm{~A}$ \\
T6 - SD & $20,2 \mathrm{~A}$ & $19,8 \mathrm{~A}$ \\
\hline Média & 21,3 & 20,2 \\
\hline CV $(\%)$ & 4,12 & 5,52 \\
\hline
\end{tabular}

* Amostragem feita na época do florescimento da cultura da soja.

TABELA 4 - Teor de água do solo $(\%)-2^{\mathrm{a}}$ amostragem**

\begin{tabular}{lcc}
\hline Tratamento & \multicolumn{2}{c}{ Profundidade $(\mathbf{c m})$} \\
\hline T1 - AD+GL & $13,6 \mathrm{~A}$ & $10-20$ \\
T2 - AA+GL & $14,5 \mathrm{~A}$ & $16,2 \mathrm{~A}$ \\
T3 - GP+GL & $14,0 \mathrm{~A}$ & $16,0 \mathrm{~A}$ \\
T4 - ES+GL & $14,4 \mathrm{~A}$ & $16,4 \mathrm{~A}$ \\
T5 - ES & $14,2 \mathrm{~A}$ & $16,1 \mathrm{~A}$ \\
T6 - SD & $14,2 \mathrm{~A}$ & $16,0 \mathrm{~A}$ \\
\hline Média & 14,3 & 16,2 \\
\hline CV $(\%)$ & 6,83 & 2,54 \\
\hline
\end{tabular}

** Amostragem feita após a colheita da cultura da soja.

Observa-se pela Tabela 5, diferença apenas na camada de 0 a $5 \mathrm{~cm}$ de profundidade para resistência do solo à penetração, podendo ser explicada pelo tráfego de máquinas e pessoas sobre a área no decorrer do experimento. 
Nota-se que a resistência do solo à penetração na semeadura direta para profundidade de $0-5 \mathrm{~cm}$ foi maior em relação aos tratamentos $\mathrm{T} 2$ e T4, os quais provocam certa mobilização na camada superior do solo aumentando sua porosidade. Pelegrin et al. (1994), Secco \& Reinert (1997) e Urchei (1996) afirmam que o plantio direto torna a camada superior do perfil do solo mais compacta, com maior densidade e com maior resistência à penetração.

Observa-se também uma diminuição do coeficiente de variação ao longo dos perfis devido à menor suscetibilidade das camadas profundas a fatores físicos incidentes diretamente no solo afetando suas características.
Na Tabela 6, os tratamentos analisados não diferiram entre si.

Analisando-se a população inicial e final da cultura da soja, pode-se observar que não houve diferença entre os tratamentos, indicado pela boa germinação da semente nos sistemas de preparo utilizados (Tabela 7).

Os dados obtidos são semelhantes aos encontrados por Coan et al. (1982) quando analisando os efeitos de diferentes preparos do solo para a cultura da soja, não encontraram diferenças significativas na quantidade de plantas por hectare e nos tratamentos grade pesada, enxada rotativa e semeadura direta.

TABELA 5 - Resistência do solo à penetração $(\mathrm{MPa})$ - $1^{\mathrm{a}}$ amostragem*

\begin{tabular}{llrrrrrrr}
\hline \multirow{2}{*}{ Tratamentos } & \multicolumn{10}{c}{$\mathbf{0 - 5}$} & $\mathbf{5 - 1 0}$ & $\mathbf{1 0 - 1 5}$ & $\mathbf{1 5 - 2 0}$ & $\mathbf{2 0 - 2 5}$ & $\mathbf{2 5 - 3 0}$ & $\mathbf{3 0 - 3 5}$ & $\mathbf{3 5 - 4 0}$ \\
\cline { 2 - 9 } & $1,22 \mathrm{AB}$ & $2,24 \mathrm{~A}$ & $3,59 \mathrm{~A}$ & $4,77 \mathrm{~A}$ & $7,30 \mathrm{~A}$ & $8,49 \mathrm{~A}$ & $8,99 \mathrm{~A}$ & $7,47 \mathrm{~A}$ \\
$\mathrm{~T} 1-\mathrm{AD}+\mathrm{GL}$ & $0,89 \mathrm{~B}$ & $2,07 \mathrm{~A}$ & $3,76 \mathrm{~A}$ & $5,11 \mathrm{~A}$ & $6,29 \mathrm{~A}$ & $8,32 \mathrm{~A}$ & $7,98 \mathrm{~A}$ & $8,82 \mathrm{~A}$ \\
$\mathrm{~T} 2-\mathrm{AA}+\mathrm{GL}$ & $1,05 \mathrm{AB}$ & $2,56 \mathrm{~A}$ & $4,94 \mathrm{~A}$ & $6,97 \mathrm{~A}$ & $8,49 \mathrm{~A}$ & $8,83 \mathrm{~A}$ & $8,49 \mathrm{~A}$ & $8,32 \mathrm{~A}$ \\
$\mathrm{~T} 3-\mathrm{GP}+\mathrm{GL}$ & $0,72 \mathrm{~B}$ & $1,73 \mathrm{~A}$ & $2,92 \mathrm{~A}$ & $5,11 \mathrm{~A}$ & $6,78 \mathrm{~A}$ & $7,47 \mathrm{~A}$ & $8,49 \mathrm{~A}$ & $8,99 \mathrm{~A}$ \\
$\mathrm{~T} 4-\mathrm{ES}+\mathrm{GL}$ & $1,05 \mathrm{AB}$ & $2,74 \mathrm{~A}$ & $3,76 \mathrm{~A}$ & $5,11 \mathrm{~A}$ & $6,78 \mathrm{~A}$ & $8,49 \mathrm{~A}$ & $9,67 \mathrm{~A}$ & $9,33 \mathrm{~A}$ \\
T5 - ES & $2,07 \mathrm{~A}$ & $3,42 \mathrm{~A}$ & $6,12 \mathrm{~A}$ & $6,97 \mathrm{~A}$ & $8,83 \mathrm{~A}$ & $8,99 \mathrm{~A}$ & $8,99 \mathrm{~A}$ & $8,99 \mathrm{~A}$ \\
T6 - SD & 1,16 & 2,46 & 4,18 & 5,67 & 7,41 & 8,43 & 8,76 & 8,65 \\
\hline Média & 42,72 & 48,59 & 48,58 & 42,30 & 35,10 & 29,40 & 21,83 & 27,65 \\
\hline CV $(\%)$ & &
\end{tabular}

* Amostragem feita na época do florescimento da cultura da soja;

TABELA 6 - Resistência do solo à penetração $(\mathrm{MPa})$ - 2a amostragem**

\begin{tabular}{|c|c|c|c|c|c|c|c|c|}
\hline \multirow{2}{*}{ Tratamentos } & \multicolumn{8}{|c|}{ Profundidade } \\
\hline & $0-5$ & $5-10$ & $10-15$ & $15-20$ & $20-25$ & $25-30$ & 30-35 & $35-40$ \\
\hline $\mathrm{T} 1-\mathrm{AD}+\mathrm{GL}$ & $1,73 \mathrm{~A}$ & $3,76 \mathrm{~A}$ & $10,68 \mathrm{~A}$ & $13,55 \mathrm{~A}$ & $14,90 \mathrm{~A}$ & $14,74 \mathrm{~A}$ & $15,74 \mathrm{~A}$ & $7,47 \mathrm{~A}$ \\
\hline $\mathrm{T} 2-\mathrm{AA}+\mathrm{GL}$ & $1,90 \mathrm{~A}$ & $7,14 \mathrm{~A}$ & $10,34 \mathrm{~A}$ & $11,02 \mathrm{~A}$ & $12,03 \mathrm{~A}$ & $10,35 \mathrm{~A}$ & $8,66 \mathrm{~A}$ & $8,82 \mathrm{~A}$ \\
\hline $\mathrm{T} 3-\mathrm{GP}+\mathrm{GL}$ & $1,39 \mathrm{~A}$ & $2,58 \mathrm{~A}$ & 7,99 A & $10,01 \mathrm{~A}$ & $10,51 \mathrm{~A}$ & $8,32 \mathrm{~A}$ & $11,02 \mathrm{~A}$ & $8,32 \mathrm{~A}$ \\
\hline $\mathrm{T} 4-\mathrm{ES}+\mathrm{GL}$ & $2,07 \mathrm{~A}$ & $2,07 \mathrm{~A}$ & $6,97 \mathrm{~A}$ & 7,98 A & $7,13 \mathrm{~A}$ & $6,63 \mathrm{~A}$ & $7,30 \mathrm{~A}$ & $8,99 \mathrm{~A}$ \\
\hline $\mathrm{T} 5-\mathrm{ES}$ & $1,56 \mathrm{~A}$ & $2,92 \mathrm{~A}$ & $8,15 \mathrm{~A}$ & $9,16 \mathrm{~A}$ & $12,37 \mathrm{~A}$ & $16,25 \mathrm{~A}$ & $14,40 \mathrm{~A}$ & $9,33 \mathrm{~A}$ \\
\hline $\mathrm{T} 6-\mathrm{SD}$ & $1,90 \mathrm{~A}$ & $5,79 \mathrm{~A}$ & $8,99 \mathrm{~A}$ & $10,68 \mathrm{~A}$ & $10,85 \mathrm{~A}$ & $11,02 \mathrm{~A}$ & $10,51 \mathrm{~A}$ & $8,99 \mathrm{~A}$ \\
\hline Média & 1,75 & 4,04 & 8,35 & 10,39 & 11,30 & 11,21 & 11,27 & 8,65 \\
\hline $\mathrm{CV}(\%)$ & 23,96 & 79,95 & 41,45 & 47,60 & 45,15 & 40,64 & 43,38 & 27,65 \\
\hline
\end{tabular}

** Amostragem feita na após a colheita da cultura da soja. 
A altura das plantas de soja aos 60 e 90 dias após a emergência não apresentou diferença entre os tratamentos analisados (Tabela 8). Resultados semelhantes foram obtidos por Bizzi et al. (2001), estudando a influência destes mesmos sistemas de preparo do solo na cultura do milho, observando que não houve diferença significativa para altura das plantas aos 30, 60 e 90 dias após a emergência, bem como para estande inicial.

Analisando-se o número de vagens por planta e grãos por vagem, observa-se não ocorrer diferenças entre os tratamentos (Tabela 9), diferindo dos resultados obtidos por Furlani (2000) em experimento com feijão, no qual o autor obteve valores diferentes para os preparos do solo com escarificador e convencional (10,6 e 9,2 vagens respectivamente).

Os valores obtidos com a produção de grãos não diferiram entre si (Tabela 10), concordando com os resultados obtidos por Benatti Júnior et al. (1984), Coan et al. (1982), Moraes \& Benez (1996), Vidigal Filho et al.
(2000) e Zaffaroni et al. (1991), que também chegaram a conclusões semelhantes quando trabalharam com milho avaliando sua produtividade em diferentes sistemas de preparo de solo.

A baixa produtividade da cultura deve-se à semeadura tardia do experimento (10 de janeiro) devida à escassez de chuvas, uma vez que nesta região, o limite máximo para o plantio da soja é a primeira quinzena de dezembro (EMBRAPA, 2003) sendo afetada pela escassez de chuva na época do florescimento, interferindo diretamente na produtividade.

Pelo observado neste trabalho pode-se concluir que, para o teor de água no solo nas profundidades de 0 10 e 10-20, não há diferença entre os tratamentos analisados. Na camada superficial do solo, a semeadura direta apresenta maior resistência à penetração na época do florescimento da cultura. As características agronômicas da cultura da soja não são influenciadas pelos tratamentos analisados.

TABELA 7 - População inicial e final das plantas de soja $\left(\mathrm{pl} \mathrm{ha}^{-1}\right)$.

\begin{tabular}{lcc}
\hline Tratamento & Estande inicial & Estande final \\
\hline T1 - AD+GL & $433.333 \mathrm{~A}$ & $377.778 \mathrm{~A}$ \\
T2 - AA+GL & $500.000 \mathrm{~A}$ & $444.444 \mathrm{~A}$ \\
T3 - GP+GL & $466.667 \mathrm{~A}$ & $377.778 \mathrm{~A}$ \\
T4 - ES+GL & $488.889 \mathrm{~A}$ & $411.111 \mathrm{~A}$ \\
T5 - ES & $433.333 \mathrm{~A}$ & $422.222 \mathrm{~A}$ \\
T6 - SD & $455.556 \mathrm{~A}$ & $422.222 \mathrm{~A}$ \\
\hline Média & 462.222 & 412.222 \\
\hline CV $(\%)$ & 12,84 & 10,92 \\
\hline
\end{tabular}

TABELA 8 - Altura da soja aos 60 e 90 dias após a emergência (cm).

\begin{tabular}{lcc}
\hline Tratamento & $\mathbf{6 0}$ dias & $\mathbf{9 0}$ dias \\
\hline T1 - AD+GL & $70,2 \mathrm{~A}$ & $101,9 \mathrm{~A}$ \\
T2 - AA+GL & $69,9 \mathrm{~A}$ & $100,9 \mathrm{~A}$ \\
T3 - GP+GL & $68,9 \mathrm{~A}$ & $99,1 \mathrm{~A}$ \\
$\mathrm{~T} 4-\mathrm{ES}+\mathrm{GL}$ & $70,0 \mathrm{~A}$ & $101,2 \mathrm{~A}$ \\
$\mathrm{~T} 5-\mathrm{ES}$ & $69,9 \mathrm{~A}$ & $98,2 \mathrm{~A}$ \\
T6 - SD & $68,8 \mathrm{~A}$ & $96,9 \mathrm{~A}$ \\
\hline Média & 69,6 & 99,7 \\
\hline CV $(\%)$ & 4,02 & 3,47 \\
\hline
\end{tabular}


TABELA 9 - Número de vagens por planta de soja e número de grãos por vagem.

\begin{tabular}{lcc}
\hline Tratamento & Vagem planta $^{-1}$ & Grão vagem $^{-1}$ \\
\hline T1 - AD+GL & $50,0 \mathrm{~A}$ & $1,70 \mathrm{~A}$ \\
T2 - AA+GL & $48,5 \mathrm{~A}$ & $1,71 \mathrm{~A}$ \\
T3 - GP+GL & $45,8 \mathrm{~A}$ & $1,69 \mathrm{~A}$ \\
T4 - ES+GL & $50,5 \mathrm{~A}$ & $1,65 \mathrm{~A}$ \\
T5 - ES & $46,9 \mathrm{~A}$ & $1,77 \mathrm{~A}$ \\
T6 - SD & $51,0 \mathrm{~A}$ & $1,70 \mathrm{~A}$ \\
\hline Média & 48,8 & 1,70 \\
\hline CV $(\%)$ & 14,63 & 5,78 \\
\hline
\end{tabular}

TABELA 10 - Produtividade de grãos em kg ha-1 .

\begin{tabular}{ll}
\hline Tratamento & Kg ha $^{-1}$ \\
\hline $\mathrm{T} 1-\mathrm{AD}+\mathrm{GL}$ & $1.430 \mathrm{~A}$ \\
$\mathrm{~T} 2-\mathrm{AA}+\mathrm{GL}$ & $1.700 \mathrm{~A}$ \\
$\mathrm{~T} 3-\mathrm{GP}+\mathrm{GL}$ & $1.485 \mathrm{~A}$ \\
$\mathrm{~T} 4-\mathrm{ES}+\mathrm{GL}$ & $1.410 \mathrm{~A}$ \\
$\mathrm{~T} 5-\mathrm{ES}$ & $1.330 \mathrm{~A}$ \\
$\mathrm{~T} 6-\mathrm{SD}$ & $1.240 \mathrm{~A}$ \\
\hline Média & 1.435 \\
\hline $\mathrm{CV}(\%)$ & 12,29 \\
\hline
\end{tabular}

\section{REFERÊNCIAS BIBLIOGRÁFICAS}

BENATTI JÚNIOR, R.; MOREIRA, C. A.; FRANÇA, G. V. Avaliação dos efeitos de sistemas de cultivo na produção de milho e nas propriedades edáficas em latossolo roxo no estado de São Paulo. Revista Brasileira de Ciência do Solo, Campinas, v. 8, n. 1, p. 139-144, 1984.

BIZZI, A. C.; REIS, G. N.; GROTTA, D. C. C.; MALASPINA, I. C.; FURLANI, C. E. A.; LOPES, A. Efeito do sistema de preparo do solo no desenvolvimento da cultura do milho (Zea mays L.). In: CONGRESSO DE INICIAÇÃO CIENTÍFICA DA UNESP, 13., 2001, Bauru. Anais... Bauru: Unesp, 2001.
BOLLER, W. Entrevista concedida à Universidade de Passo Fundo, RS. 2003. Disponível em: http://Www.upf. bry Acesso em: 18 nov. 2003.

COAN, O.; ORTOLANI, A. F.; BANZATTO, D. A. Efeitos de diferentes sistemas de preparo do solo na produção da soja. Revista Brasileira de Engenharia Agrícola, Botucatu, v. 6, n. $1,1982$.

EMPRESA BRASILEIRA DE PESQUISA AGROPECUÁRIA. Sistema brasileiro de classificação de solos. 2003. Disponível em: 〈http://Www.cnpso.embrapa.bri >. Acesso em: 11 nov. 2003.

EMPRESA BRASILEIRA DE PESQUISA AGROPECUÁRIA. Centro nacional de pesquisas de solos. Sistema brasileiro de classificação de solos. Brasília, DF, 1999. $412 \mathrm{p}$.

FURLANI, C. E. A. Efeito do preparo do solo e do manejo da cobertura de inverno na cultura do feijoeiro (Phaseolus vulgaris L.). 2000. 218 f. Tese (Doutorado em Energia na Agricultura) - Universidade Estadual Paulista, Botucatu, 2000.

MORAEZ, M. H.; BENEZ, S. H. Efeitos de diferentes sistemas de preparo do solo em algumas propriedades físicas de uma Terra Roxa estruturada e na produção de milho para um ano de cultivo. Revista Engenharia Agrícola, Botucatu, v. 16, n. 2, p. 31-41, 1996.

PELEGRIN, F.; MORENO, F.; MARTIN-ARANJA, J.; FERNANDEZ, G. P. Influencia del sistema de laboreo em la resistencia a la penetracion y otras propiedades fisicas del suelo. Investigacion Agricola, Santiago, v. 11, p. 409-425, 1994.

SECCO, D.; REINERT, D. J. Efeito imediato e residual de escarificadores em Latossolo Vermelho Escuro sob plantio direto. Engenharia Agrícola, [S.1.], v. 16, n. 3, p. 52-61, 1997.

STOLF, R. Fórmulas de transformação dos dados de penetrômetro de impacto em força/unidade de área. In: CONGRESSO BRASILEIRO DE ENGENHARIA AGRÍCOLA, 19., 1990, Piracicaba. Anais... Piracicaba: Sociedade Brasileira de Engenharia Agrícola, 1990. v. 2, p. 823-837. 
STOLF, R.; FERNANDES, J.; FURLANI NETO, V. L. Recomendação para uso do penetrômetro de impacto Modelo IAA/Planalsulcar-Stolf: Ministério da Indústria e do Comércio: Programa Nacional de Melhoramento da Cana-de-Açúcar - Planalsucar. Brasília, DF: Ministério da Indústria e do Comércio, 1983.

URCHEI, M. A. Efeitos do plantio direto e do preparo convencional sobre alguns atributos físicos de um Latossolo Vermelho-Escuro argiloso e no crescimento e desenvolvimento do feijoeiro (Phaseolus vulgaris L.) sob irrigação. 1996. 131 f. Tese (Doutorado em Irrigação e Drenagem)-Universidade Estadual Paulista, Botucatu, 1996.
VIDIGAL FILHO, P. S.; PEQUENO, M. G.; SAGRILO, E.; TORMENA, C. A.; KVITSCHAL, M. V. Efeitos de sistemas de preparo na cultura do milho e em algumas propriedades físicas do solo. In: CONGRESSO NACIONAL DE MILHO E SORGO, 23., 2000, Uberlândia, MG. Anais... Uberlândia: UFU, 2000.

ZAFFARONI, E.; BARROS, H. H.; NÓBREGA, J. A. M.; LACERDA, J. T.; SOUZA JÚNIOR, V. E. Efeito de métodos de preparo do solo na produtividade e outras características agronômicas de milho e feijão no nordeste do Brasil. Revista Brasileira de Ciência do Solo, Campinas, v. 15, n. 1, p. 99104, 1991. 